\title{
La réécriture d'un texte hagiographique au XIIe siècle : la Vita sancti Sansonis, de Baudri de Bourgueil
}

\section{Armelle Le Huërou}

\section{(2) OpenEdition \\ Journals}

Édition électronique

URL : http://journals.openedition.org/abpo/1729

DOI : $10.4000 /$ abpo. 1729

ISBN : 978-2-7535-1482-9

ISSN : 2108-6443

\section{Éditeur}

Presses universitaires de Rennes

Édition imprimée

Date de publication : 20 juin 2001

Pagination : $7-30$

ISBN : 978-2-86847-635-7

ISSN : $0399-0826$

\section{Référence électronique}

Armelle Le Huërou, «La réécriture d'un texte hagiographique au XIle siècle : la Vita sancti Sansonis, de Baudri de Bourgueil », Annales de Bretagne et des Pays de l'Ouest [En ligne], 108-2 | 2001, mis en ligne le 20 juin 2003, consulté le 02 mai 2019. URL : http://journals.openedition.org/abpo/1729 ; DOI :

$10.4000 / a b p o .1729$ 


\title{
La réécriture d'un texte hagiographique au XII ${ }^{\mathrm{e}}$ siècle: la Vita sancti Sansonis ${ }^{\text {, }}$ de Baudri de Bourgueil
}

\author{
Armelle LE HUËROU \\ Doctorante, CRHISCO, Université Rennes 2
}

Si Baudri de Bourgueil (1045/6-1130) a acquis une certaine renommée, il le doit moins à sa carrière ecclésiastique, pourtant prestigieuse (entré au monastère de Saint-Pierre-de-Bourgueil à une date inconnue, il en est élu abbé entre 1078 et 1082, avant d'être nommé, en 1107, archevêque de Dol), qu'à son œuvre littéraire ${ }^{2}$. Quoiqu'il soit essentiellement connu pour son œuvre poétique, il nous intéresse ici au double titre d'hagiographe de saint Samson et d'archevêque de Dol. Malheureusement pour lui, il ne portait pas particulièrement dans son cœur le pays où il dut exercer son ministère: les écrits postérieurs à son élection à la tête du clergé

1. Le texte de la Vita Sansonis nous a été conservé intégralement par un seul manuscrit (dont nous avons proposé une édition dans notre DEA): Paris, BN lat. 5350 (f $\left.{ }^{\circ} 1-105\right)$. C'est un manuscrit luxueux de format $21 \times 31 \mathrm{~cm}$, composé de 106 fol. qui date du début du XIVe siècle. Deux autres manuscrits proposent des extraits de la Vita Sansonis de Baudri: Paris, BN fr. 22321 ( $\mathrm{f}^{\circ}$ 788-793), copie tardive et hétéroclite effectuée au XVIII $\mathrm{e}$ siècle par les Mauristes et fait partie de la collection des Blancs-Manteaux et Avranches, $\mathrm{BM}, \mathrm{ms} 168\left(\mathrm{f}^{\circ} 68 \mathrm{v}^{\circ}-71\right)$ qui propose un texte très abrégé et découpé en 8 lectiones.

2. On a l'habitude de considérer que la production littéraire de Baudri s'articule en deux grands ensembles: les textes poétiques, composés dans leur grande majorité à Bourgueil et les textes en prose qui coïncident plutôt avec la période «bretonne» de sa vie. Ces textes sont aujourd'hui très inégalement étudiés et édités, puisque, à ce jour, seuls les premiers ont fait l'objet de travaux récents dont un des principaux promoteurs est M. Jean-Yves Tilliette, qui a consacré à Baudri poète de nombreux articles et a donné récemment l'édition et la traduction du premier volume de ses Carmina (Carmina, t.1, éd., trad. et comm. Jean-Yves Tilliette, Paris, Les Belles Lettres, 1998. Le second volume est à paraitre prochainement, et nous remercions beaucoup M.Tilliette de nous en avoir communiqué gracieusement le contenu). Les seconds, quant à eux, revêtent une grande diversité générique. Nous sont ainsi parvenus deux Lettres (Epistula ad Fiscannenses, Epistula ad Petrum Gemmeticensum priorem), un petit traité spirituel (De visitatione infirmorum), deux ouvrages "historiques", réécritures de textes antérieurs (Gesta pontificum Dolensium, Historia hierosolymitana), trois Vitae (Vita beati Roberti de Arbrissello, Vita sancti Hugonis Rotomagensis episcopi, Vita sancti Samsonis) et deux textes apparentés (Acta translationis capitis sancti Valentini martyris Gemmeticum in Gallia, De scuto et gladio sancti Michaelis). 
breton sont à ce sujet explicites. Il se plaint auprès de Pétronille de Chemillé (dans le prologue de sa Vie du bienheureux Robert d'Arbrissel) de vivre "au milieu des scorpions" en Petite Bretagne, d'y être en bute à "la férocité bestiale ${ }^{3}$ ». Et quand, dans la Vie de saint Samson, sous prétexte de dépeindre les Bretons de l'époque du héros, il souligne à plaisir la barbarie et la bêtise crasse qui sévissent en Bretagne, il y a tout lieu d'y voir une caricature sournoise de ses contemporains ${ }^{4}$. Il est bien sûr difficile, comme toujours chez Baudri - et chez la plupart de ses contemporains de faire la part entre ce qui relève du cliché (les textes s'accordent unanimement à présenter une image exagérément noircie de la Bretagne et de ses occupants ${ }^{5}$ ) et ce qui relève d'un réel sentiment de mépris, voire d'aversion. Mais Baudri avait des raisons valables de ne pas apprécier ses administrés, ce qui donne à la répulsion qu'il professe à leur endroit un accent de sincérité. Au départ, son élection, pour canonique qu'elle soit, est une élection par défaut : le clergé de Dol avait préféré le chancelier de l'église de Chartres, mais celui-ci, soutenu par son archevêque (Yves de Chartres), se désista. Ensuite, les péripéties qui marquent son épiscopat ont dû contribuer à maintenir des relations conflictuelles entre Baudri et son clergé: suite à un différend avec son chapitre, il est suspendu temporairement par le légat pontifical et ses suffragants ne le soutiennent pas unanimement (loin s'en faut) dans la querelle qui oppose la métropole de Dol à Tours ${ }^{6}$. Il est même avéré que Baudri choisit de séjourner fréquemment dans les enclaves normandes de Dol (il est d'ailleurs enterré à Préaux), qu'il multiplia les visites en Grande-Bretagne ou en Normandie. Cet exil volontaire, Orderic Vital, qui se vante d'avoir bien connu Baudri, l'impute explicitement à la "perversité» des Bretons, que l'archevêque "ne pouvait supporter»: "C'est pourquoi il fuyait souvent leur insolence et leur dérèglement et se réfugiait en Normandie où l'Église de Dol avait des possessions sur la rivière Risle [...]. Là, par ses écrits et ses enseignements, il incitait ses auditeurs à honorer Dieu et il se rendait fréquemment en visite dans les monastères voisins de Fécamp, Fontenelle, Jumièges ${ }^{7}$ ". Par ailleurs, la Bretagne d'alors, stigmatisée pour l'ignorance et la grossièreté

3. Migne, Patrologie Latine (P.L.), t.166, col. 1045, prol., 2: «[...] cum me et multa mundi fluctivagi inquietet procella, et maxime minoris Britanniae, in qua cum scorpionibus habito, bestialis, geminaque circumvallit ferocitas".

4. C'est ainsi par exemple que, au début du livre II (1, $\left.\mathrm{f}^{\circ} 67\right)$, Baudri évoque l'œuvre pastorale de Samson aux alentours de Dol en ces termes: "Exibat [...] ad circumsitas ciuitates regionesque tum ut barbaras gentes uisitaret, tum ut uerbum Dei [...] beluinis et indisciplinatis hominibus ministraret" (il sortait pour aller dans les cités et régions voisines, aussi bien pour rendre visite aux populations barbares que pour administrer la parole de Dieu [...] à des hommes bêtes et déréglés).

5. Sur la notion de cliché, on se référera à l'article de Pierre RICHE: «Les bretons victimes des lieux communs dans le haut Moyen Âge», LE MEnN, Gwénolé et LE MoING, Jean-Yves, (éd.), Bretagne et pays celtiques, langues, histoire, civilisation. Mélanges offerts à la mémoire de Léon Fleuriot, Saint-Brieuc-Rennes, 1992, p.111-115.

6. Cf. Duine, F., La métropole de Bretagne, Champion, Paris, 1916, p.35-36 et p.118.

7. Orderic Vital, Historia Aecclesiastica, vol. V, (IX, 18), éd. M. Chibnall, Oxford, 1975, trad. Armelle Le Huërou. 
de ses habitants, clercs et laïcs confondus, devait logiquement déplaire au lettré raffiné qu'était Baudri, sous peine de renier son statut de poète reconnu et apprécié, de membre actif de la "Renaissance» du $\mathrm{XII}^{\mathrm{e}}$ siècle.

Malgré ces déboires, Baudri semble avoir assumé les devoirs liés à sa charge de manière tout à fait convenable, sinon irréprochable - dès lors que l'on excepte ses trop fréquents séjours hors de son archevêché. Il s'est même employé à donner une version rénovée de la vie de son illustre prédécesseur sur le siège archiépiscopal de Dol. Évidemment, le Samson historique ${ }^{8}$ n'a jamais été archevêque, mais l'essentiel est que Baudri en soit convaincu et qu'il s'attelle au remaniement d'une Vita sansonis carolingienne ${ }^{9}$, elle-même réfection d'un texte antérieur ${ }^{10}$.

Baudri, conformément aux lois du genre, précise dans un prologue ${ }^{11}$ les raisons qui ont motivé son entreprise. Si l'on en croit l'archevêque, l'intérêt qu'il porte à Samson est le pur fruit de la nécessité: il faut rivaliser avec les œuvres littéraires des "Gentils» qui entretiennent le souvenir de leurs philosophes. Leurs livres submergent la littérature, encombrent les mémoires chrétiennes. Conséquence logique de ce constat: les chrétiens doivent émerger de leur torpeur, se mettre à l'ouvrage et exalter leurs glorieux représentants ${ }^{12}$. C'est donc en toute légitimité que Baudri se propose, sous la férule de l'Esprit saint, de révéler au grand jour le caractère exemplaire de Samson ${ }^{13}$.

Il reste malgré tout un point à élucider: pourquoi Samson en particulier? Baudri est d'ordinaire un hagiographe commandité qui, comme tel, travaille sur sujet imposé, et n'a pas à s'étendre sur ses motivations et

8. Samson, dont la présence au troisième concile de Paris (563) semble bien attester la qualité d'évêque, n'a bien sûr jamais été métropolitain (Dol, comme tous les évêchés bretons dépendent alors incontestablement de Tours), encore moins archevêque, puisque ce terme n'apparaît qu'avec saint Boniface (VIII ${ }^{\mathrm{e}}$ siècle).

9. Le texte de cette Vita anonyme, qu'on a l'habitude d'appeler Vita Secunda, (en référence à son modèle plus ancien, la Vita Prima), est édité par Dom Plaine: «Vita antiqua sancti Samsonis", Analecta Bollandiana, 6, 1887, p.77- 150.

10. Il s'agit de la fameuse Vita armoricaine la plus ancienne, dont M. Pierre Flobert a donné il y a peu une édition (FLOBERT, Pierre, La vie ancienne de saint Samson de Dol, CNRS éditions, 1997) et dont la date de rédaction reste controversée par les spécialistes. Voir état de la question dans Flobert, ibid., p.102-111.

11. Dom Plaine (D. P.) en propose le texte (avec quelques inexactitudes) dans le cadre de son édition de la Vita IIa., p.80-82 (cf. supra note 9).

12.Prol. $\left(\mathrm{f}^{\circ} 1 \mathrm{r}^{\circ}-\mathrm{v}^{\circ}\right)$ : “Cum gentilium phylosophos meminerimus multos suorum laudibus pomposis extulisse, et super hoc libros litteris aureis rutilantes edidisse, quid nos tocius secordie a Christi laude ex hoc nunc tacebimus redarguendi adhuc torpemus? Si illi per ambages suas friuolas homines impuros et perditos celis intulerunt, et posteritatis memorie taliter eos commendauerunt, cur Deum nostrum in sanctis suis mirabilem non predicare et non glorificare non erubescimus? Expergiscamur igitur aliquando et ad capescendam illam rem publicam specialiter gloriosam accendamur, et saltem orthodoxorum exemplis incitemur $[\ldots]$ "...

13. Idem ( $\left.{ }^{\circ} 2\right)$ : "In confessorum igitur aliquantulum demorantes collegio domnum Sansonem ad medium deducamus, et qualiter Deo suo complacuerit [...] ad auditorum utilitatem stili ueridici censura recitemus." 
objectifs: il lui suffit d'évoquer les commanditaires ${ }^{14}$ - qui sont aussi généralement les destinataires du texte. Ici, c'est apparemment de sa propre initiative qu'il s'est mis à l'œuvre, et, si l'on s'en tient à ses déclarations liminaires, c'est d'abord dans un but moral et pédagogique. Mais, malgré les épithètes hyperboliques dont Baudri prend soin de gratifier Samson, "le lys le plus éclatant parmi les confesseurs", "parmi les prêtres l'encens et l'encensoir, l'officiant et le sacrifice ${ }^{15}$ ", on voit mal en quoi ce dernier, par sa seule personnalité, aurait suscité une telle vocation chez son biographe... Si l'on additionne tous ces éléments (le manque d'explications satisfaisantes sur le choix de Samson comme parangon de sainteté, l'absence de commanditaire, le silence sur d'éventuels destinataires précis) à la position de Baudri, héritier de Samson puisque archevêque, et à son insistance à afficher une ambition exclusivement morale et didactique, tout laisse supposer des raisons d'ordre pratique: l'autorité métropolitaine de Dol, très contestée, pour ne pas dire niée, et celle, souvent mise à mal, de son actuel archevêque avaient sans doute elles aussi besoin d'être restaurées et réhabilitées. Il est donc très plausible que Baudri "se proposa particulièrement d'établir, au moins par la possession, son prétendu droit de métropolitain de Bretagne, qu'il fait remonter à saint Samson ${ }^{16}$ ", mais il semble que Baudri se soucie davantage encore de Pental, cette enclave doloise dont il consacra l'église un mois avant sa mort ${ }^{17}$.

Quoique cette question demeure cruciale, la manière dont le texte est (re)pensé, (re)construit et (re)écrit est riche d'enseignements. Le soin extrême apporté par Baudri à la réfection strictement littéraire témoigne en effet d'une réflexion sur la nature et la finalité du texte hagiographique. Objet littéraire en même temps qu'instrument d'évangélisation, il se doit d'offrir des qualités formelle et esthétique irréprochables qui contribuent pleinement à sa force de persuasion et de conviction. Cela dit, pour fonctionner efficacement, le discours hagiographique, si séduisant soit-il, doit impérativement s'ancrer dans la réalité de l'auditoire à qui il est destiné, en lui proposant un texte adapté, non seulement à ses goûts littéraires, mais aussi à sa mentalité.

14. Voir par exemple, la Vita Beatissimi Roberti de Arbrisello, P.L. t.162, col.1043 sq ou Vita sancti Hugonis, P.L., t.166, col.1163-4.

15. Prol.( $\left(\mathrm{f}^{\circ} 2 \mathrm{v}^{\circ}\right)$ : « inter confessores lilium conspectissimum ». Idem (f $\left.{ }^{\circ} 3\right)$ : " qui summo sacerdotum fuit thus et thymiamaterium, sacerdos et sacrificium ".

16. Histoire littéraire de la France, t.XI, p.103, reproduit dans P.L., t.166, col.1053-4.

17. Nous ne nous appuyons pour l'instant que sur des éléments pour la plupart internes au texte, mais il apparaît que l'importance accordée à Pental (tant dans la définition de son statut de "second siège" (" secunda sedes ", $\mathrm{f}^{\circ} 81$ ) de Dol que dans le traitement particulier des miracles qui s'y déroulent) par le texte est capitale. Par ailleurs, le fait que le manuscrit propose le texte de la Vita Sansonis suivi des hymnes que Baudri composa pour la consécration de l'église de Pental et le témoignage d'Orderic Vital (cf. supra, p.2 et n.7) sur l'activité pastorale de Baudri en Normandie vont également dans ce sens. Nous reviendrons sur cette question dans la thèse que nous avons entreprise sur L'œuvre hagiographique de Baudri de Bourgueil. 


\section{Une poétique de l'ornementation}

\section{«Une éloquence plus brillante»}

Les déclarations liminaires de Baudri ont le mérite d'être explicites: son travail de réfection est formel, strictement formel même, à ce qu'il prétend. Les exigences esthétiques et littéraires contemporaines sont incompatibles avec ce que contiennent les "vieux feuillets» qu'il a exhumés. Il a donc pris sur lui de substituer à "la pauvreté du style» de son modèle une "éloquence plus brillante" en adéquation avec la "matière noble et glorieuse» qu'est la vie de Samson. Il a, nous dit-il, remis au goût du jour un sujet injustement maltraité par un prédécesseur incompétent ${ }^{18}$. Il s'est contenté de le débarrasser de ses "simples haillons" pour lui fournir un aspect plus convenable, tant il est vrai que «la matière, quelle qu'elle soit, est de moindre prix, quand ne la rehausse pas l'homme rompu à la maîtrise de son art ${ }^{19}$ ". Point de salut sans artifice, nous dit donc Baudri! Il faut comprendre par là que, sans sacrifier pour autant «l'authenticité sans mélange de la matière ${ }^{20}$ », il s'est employé à remédier aux imperfections formelles de son modèle.

Baudri a donc bouleversé certaines données du texte antérieur en supprimant ou condensant, en ajoutant ou développant, mais il a choisi de maintenir le cadre dans lequel se déroule la Vita IIa : répartition du texte en deux livres (le premier correspond à la partie insulaire de la vie de Samson, le second à la partie continentale), découpage en chapitres, même nombre de chapitres pour chaque livre (20 pour le livre I, 26 pour le livre II), titres similaires pour les mêmes chapitres. C'est seulement à l'intérieur du cadre qu'il a reproduit fidèlement qu'ont lieu les transformations.

Dans un premier temps, Baudri conserve donc la charpente initiale, mais il s'attache à en consolider élégamment les différentes parties à grand renfort de transitions plus ou moins subtiles. Ainsi, à la charnière des livres I et II, là où son modèle rompait sèchement ${ }^{21}$, Baudri ménage un pont entre les deux livres, entre les deux pays auxquels chacun est consacré en se mettant en scène effectuant la traversée maritime en compagnie de Samson qui se rend de Grande en Petite Bretagne: «Maintenant donc, refermons le premier livre pour faire voile en compagnie de Samson qui navigue vers la petite Bretagne ${ }^{22}$ ». Ce qui importe ici c'est autant la navigation historique de Samson - sur laquelle on n'en saura pas plus -

18. Prol. ( $\mathrm{f}^{\circ} 2 \mathrm{v}^{\circ}$ et 3$)$ : "Erat [...] usque ad nos illa nobilis et gloriosa materia simplicibus pannis inuoluta, et ut adhuc in ueteribus si forte supersunt dinosci potest scedulis uilibus in siliquis uber granum uiluerat. Nos igitur [...] Sansonem beatissimum [...] eloquio celebriori, sed tamen ueraci proferemus, et [...] dictorum simplicitatem aliquatenus poliemus".

19. Prol. (f ${ }^{\circ}$ ): "Minus celebrioris siquidem precii est, quecumque materia, quam non exornat callens artificis pericia."

20. Prol. $\left(\mathrm{f}^{\circ} 2 \mathrm{v}^{\circ}\right)$ : "materiei mera sinceritate".

21. Vita IIa, op. cit., p.116 et 118. 
que le passage qu'elle permet d'accomplir métaphoriquement et de manière convenue d'un livre à l'autre. Et, bien sûr, à l'intérieur de chaque livre, un soin semblable - même si l'effet obtenu n'est pas toujours aussi réussi - est apporté, afin que le texte glisse sans heurt brutal d'un chapitre à l'autre. Ce souci de la forme est tout à fait représentatif des préoccupations de Baudri et, quoique, ou parce que, au détour d'une phrase, il déplore son incapacité à rivaliser avec l'éloquence d'augustes Anciens ${ }^{23}$, son acharnement à exploiter les ressources de l'arsenal rhétorique apparaît comme une tentative (pas toujours couronnée de succès) du disciple pour dépasser ses maîtres. Il serait trop fastidieux, et surtout inutile, de dresser la liste exhaustive des figures de style qu'il met à contribution, car, même s'il lui arrive de plaquer gratuitement des formules rhétoriques, le plus souvent, c'est insérées dans un contexte précis qu'elles font sens.

Du fait de sa position liminaire, le prologue est une bonne vitrine de quelques-uns des outils rhétoriques utilisés constamment par Baudri: on y trouve, dès l'attaque du texte, les classiques questions oratoires ${ }^{24}$ : virulentes, elles simulent l'indignation et sont destinées à motiver Baudri (et peut-être aussi ses confrères) ${ }^{25}$. Stigmatisant "la torpeur" qui l'(es) "engourdit", elles sont immédiatement relayées par une injonction qui poursuit la métaphore ${ }^{26}$ : "Réveillons-nous donc! ${ }^{27}$ ". Peu après cet éclat initial, Baudri se définit, en toute fausse modestie, par rapport à Samson, par un réseau d'oppositions: d'un côté "Baudri, le pontife très humble de la sainte Église de Dol», de l'autre «lui, qui fut le métropolitain de [Dol] et qui s'illustra comme archevêque de toute la Bretagne citérieure ${ }^{28}$ ". On voit le jeu qui consiste à suggérer l'humilité (revendiquée) de Baudri par rapport à son prédécesseur dans la dissymétrie lexicale (Dol / toute la Bretagne, pontife très humble/ métropolitain + archevêque) sciemment employée pour qualifier la même fonction au même

22. I, $20\left(\mathrm{f}^{\circ} 59 \mathrm{r}^{\circ}\right.$ et $\left.\mathrm{v}^{\circ}\right)$ : "Iam nunc igitur priorem librum claudentes cum Sansone in minorem Britanniam nauigante nauigemus [...]".

23. On peut, par exemple, se référer à la Vita Sancti Hugonis (P. L., t.162, prol., col. 1164), à la Vita Beati Roberti de Arbrissello (P.L., t.166, prol. 2, col. 1045) ainsi qu'à l'Historia Hierosolymitana (P.L., t.162, prol., col. 1063) où sont explicitement mentionnés Cicéron, Virgile et Salluste.

24. Prol. ( $\mathrm{f}^{\circ} 1 \mathrm{r}^{\circ}$ et $\left.\mathrm{v}^{\circ}\right)$ : "[...], quid nos tocius secordie si a Christi laude ex hoc nunc tacebimus redarguendi adhuc torpemus? [...] cur Deum nostrum in sanctis suis mirabilem non predicare et non glorificare non erubescimus?"

25. Il est difficile de trancher: rien de concluant ne permet de considérer le "nous» qui parcourt tout le premier paragraphe plutôt comme une déclinaison emphatique du «je» que comme l'expression d'une collectivité (la communauté des Chrétiens qui écrivent) à laquelle appartient Baudri.

26. La métaphore est d'ailleurs particulièrement représentée dans le prologue: le texte remanié est d'abord assimilé à un personnage aux vêtements en lambeaux, puis, comme pour préciser cette première image, à un germe fertile en train de moisir dans une cosse pourrie.

27. Prol. $\left(\mathrm{f}^{\circ} 1 \mathrm{v}^{\circ}\right)$ : "Expergiscamur igitur aliquando $[\ldots]$ ".

28. Prol. ( $\left.f^{\circ} 2\right)$ : "[...] ego Baldricus sancte Dolensis ecclesie pontifex humilimus, cuius sedis et ipse fuit metropolitanus, et tocius Britannie citerioris claruit archiepiscopus ». 
endroit. On peut également relever quelques hyperboles (dont nous avons déjà parlé) ainsi qu'un jeu sur l'homonymie qui définit saint Samson par opposition au Samson biblique dans une belle antithèse: «Parlons donc de saint Samson, non pas Samson le soldat le plus fort physiquement qui arracha la gueule du lion ${ }^{29}$, mais Samson le soldat le plus courageux moralement $[\ldots]^{30}$ ».

\section{L'emploi concerté des références scripturaires}

Cette définition de saint Samson par opposition à son homonyme vétéro-testamentaire est formellement irréprochable, mais c'est à un tout autre titre qu'elle mérite qu'on s'y arrête. Elle est beaucoup moins convenue qu'il n'y paraît: en dépit de l'homonymie et des circonstances similaires qui président à la naissance extraordinaire des deux Samson ${ }^{31}$, le rapprochement n'est jamais fait entre les deux personnages dans la Vita carolingienne à l'origine du texte de Baudri ni même dans la Vita prima. Quant à Baudri, il s'en tient seulement à cette antithèse, sans plus exploiter le parallèle entre la figure biblique et le protagoniste de sa Vita. Mais, et c'est le plus curieux, en caractérisant son protagoniste comme l'antithèse du personnage biblique, Baudri déroge aux règles suivies par les hagiographes en général - qui présentent plutôt le saint comme l'héritier direct, voire la réincarnation du personnage biblique auquel il est comparé ou, inversement, le personnage biblique comme la préfiguration du héros - en même temps qu'il s'inscrit en faux contre le traitement que luimême fait subir aux références scripturaires dans sa Vita.

En effet, si Baudri, comme tout hagiographe digne de ce nom, manipule avec une grande aisance le matériau biblique, il en fait surtout un des instruments de la refonte formelle du texte carolingien. Pour ne prendre qu'un exemple, lors de deux interventions miraculeuses de Samson pour faire jaillir de l'eau, la Vita IIa évoquait très conventionnellement Moïse ${ }^{32}$. Le texte de Baudri, en plus de reprendre à son compte ces allusions en les adaptant ${ }^{33}$, convoque cette même figure biblique dans des contextes différents: entre autres, on apprend au détour d'une phrase, que deux frères hostiles à Samson ont été "aveuglés complètement", par

29. Allusion à l'exploit de Samson relaté en Juges 14, 6 .

30. Prol. ( $\mathrm{f}^{\circ} 3$ ): «Dicemus igitur sanctum Sansonem non illum qui discerpsit ora leonis fortissimum, sed illum dumtaxat qui zabulum deuicit militem strenuissimum [...]". On retrouve également cette référence au Samson biblique en I, 3 ( $\mathrm{f}^{\circ}$ 9): «Ipse Sanson uocabitur; maior erit equidem sanctitate quam ille de quo scriptum est fuit virium fortitudine."

31. Dans les deux cas, il s'agit de fils "miraculeux": conçus sur le tard par des femmes réputées stériles, leur naissance est annoncée par un ange.

32. D.P., p.111 (I, 17): Samson et les frères viennent de se restaurer mais n'ont rien pour assouvir leur soif: "[fratres] deprecantur, ut ipse suis famulis aquam tribueret, qui Israeliticis populis in Choreb fontem dederat in heremo. In his verbis maxime sanctus Sanson supplex erat in precibus, Dominum supplicans ut preces illorum exaudiri mererentur" et D.P., p.131-132 (II, 10) où Samson, en visite chez saint Germain d'Auxerre, pour 
Dieu qui, jadis, "endurcit le cœur de Pharaon ${ }^{34}$ ", ou encore que Samson, assistant au combat qui oppose son protégé Judual à l'usurpateur Commore, "à l'instar de Moïse élevait inlassablement les mains vers le ciel ${ }^{35}$ ", à quoi on peut ajouter ce que dit Samson lui-même, pour expliquer son refus de se rendre auprès de son père malade ${ }^{36}$ : «J'ai quitté l'Égypte, grâce à Dieu, et je ne retournerai jamais auprès des marmites de viande». Les emprunts bibliques, passages obligés, permettent à Baudri de se livrer à un des exercices de virtuosité dont il est friand. L'ajout et le renouvellement des références bibliques sont peut-être les moyens triviaux de s'acquitter de son travail d'hagiographe, mais ce n'est qu'accessoirement: Baudri ne perd jamais de vue qu'il entend démontrer "avec éloquence ${ }^{37}$ " son talent "d'homme rompu à la maîtrise de son art ${ }^{38}$ "! Les allusions et réminiscences scripturaires ne sont donc pas employées de façon aléatoire, mais elles sont solidaires les unes des autres. C'est au sein des combinaisons auxquelles elles sont assujetties qu'elles prennent tout leur sens et contribuent notamment à façonner le personnage de Samson, à asseoir confortablement la légitimité de sa sainteté. Enfin, l'organisation des citations bibliques en ensembles cohérents et signifiants offre surtout un aperçu précieux de l'intérêt que Baudri porte à l'élaboration de réseaux d'images en général.

accélérer le repas qu'on n'a pas encore commencé parce que le frère chargé d'aller chercher de l'eau n'est pas rentré, propose: «Si aqua in prope non est, Dominum Creatorem omnium una mecum invocate, ut, sicut Israhelitico populo siti deficienti de petra super verticem montis Horeb aquam tribuere dignatus est, ita modo meritis vestris fontem aquae in perpetuum manantem reddere dignetur." À la suite de quoi, de l'extrémité de son bâton, il touche une pierre d'où jaillit un cours d'eau. Les deux épisodes se réfèrent à Ex. 17, 1-7.

33. Dans le premier cas (I, $17\left(f^{\circ} 51 v^{\circ}\right)$ ), le parallèle entre les deux personnages est lisible dans la construction même de la phrase: «Lui qui à l'époque de Moïse fit couler l'eau du rocher en abondance, voici qu'aux jours où vivait Samson Il eut soin des disciples en prières et leur fournit l'occasion appropriée d'apaiser leur soif". Dans le second cas (II, 10 ( $\left.\mathrm{f}^{\circ} 82\right)$ ), après une intervention orale sensiblement identique à celle prononcée dans la Vita IIa ("Invoquons, frère Germain, le Seigneur Dieu qui dans le désert de Sinai a fait jaillir l'eau de la roche pour le peuple israélite. Qu'ici aussi, Il nous arrose de Sa bénédiction qui coule à flots, afin que nous nous félicitions de ses bienfaits et que nous assouvissions notre soif à la fontaine de sa grâce"), Samson accomplit les mêmes gestes que Moïse, ce que souligne l'emploi des expressions bibliques: "Il prononça ses paroles et, de la pointe de son bâton, frappa la large pierre $(P S .77,20,2)$ qui jouxtait le pressoir: il fit jaillir l'eau ( $P$ s. $77,16,22)$ qui, aujourd'hui encore, coule en un très large ruisseau".

34. I, $8\left(f^{\circ} 16 \mathrm{v}^{\circ}\right)$ : "[...] eos excecauerat, Qui dudum induruit cor Pharaonis", qui renvoie à Ex. 9, 12 (" induravitque Dominus cor Pharaonis "), et également à Ex.7, 13; 7, 22; 8, 32, etc.

35. II, 17 ( $\left.\mathrm{f}^{\circ} 95\right)$ : "Sanson etiam, cum Moise, manus in celum indefessus leuabat [...]", allusion au combat d'Israël contre Amaleq (Ex. 17, 11: "cumque leuaret Moses manus, vincebat Israhel; sin autem paululum remisisset, superabat Amalech ").

36. I, 9 ( $\left.\mathrm{f}^{\circ} 24 \mathrm{v}^{\circ}-25\right)$ : «Egyptum, Deo gratias, aufugi; ad ollas carnium numquam reuertar». Pour les réminiscences bibliques: Deuter. 5, 15 et Ex. 16, 3.

37. Prol. ( $\left.\mathrm{f}^{\circ} 3\right)$ : « eloquio ".

38. Id.: "callens artificis pericia ». 


\section{Réseaux d'images et jeux sur les mots}

Il semble en effet se faire une loi de multiplier les jeux sur les images et les mots tout en disséminant les éléments de compréhension: une expression anodine, une métaphore plus ou moins usée se révèlent souvent moins faciles à saisir qu'on ne pourrait le penser d'abord, parce qu'il faut réunir des éléments épars, confronter des passages entiers pour en faire émerger un contenu latent. L'interprétation d'une petite expression, " uir columbinus ", qui ne se fait pas remarquer par son originalité et qui est utilisée en deux endroits du texte ${ }^{39}$ pour désigner Samson, résume assez bien notre propos. La première occurrence semble transparente, même si elle joue sur la polysémie de l'adjectif. Comme Baudri a pris soin de préciser plus haut que Samson "alliait la prudence du serpent et la simplicité de la colombe ${ }^{40}$ ", ménageant ainsi une transition, sinon subtile du moins habile, entre le chapitre 6 et le 7, où l'ordination de Samson comme diacre se déroule en présence d'une colombe, le sens du jeu avec les mots auquel il se livre lors de la seconde apparition de la colombe paraît évident: «Et cette fois encore [lors de son ordination au sacerdoce], trois témoins vénérables ne manquèrent pas de voir, comme la première fois pendant son ordination [comme diacre], une colombe descendre au-dessus de sa tête et y rester et ils l'attestèrent ensuite dans un témoignage sur lequel il est impossible de jeter le discrédit. Ne convenait-il pas que l'homme colombin (vir columbinus) fût désigné par la colombe et qu'il fût voué à cette colombe qui, selon le témoignage de Jean, resta tranquille audessus du Christ dans le Jourdain? (I, 8) ${ }^{41}$. "Vir columbinus" renvoie à l'incarnation par Samson du précepte évangélique énoncé en I, 6, à quoi le contexte immédiat, incluant l'expression dans une scène où la colombe est, pour la seconde fois, la manifestation de la présence de l'Esprit saint, ajoute une autre dimension: la confirmation de la "cohabitation" intime de l'Esprit saint avec Samson ${ }^{42}$. En revanche, quand on rencontre à nouveau l'expression (II, 16), le passage dans lequel elle est employée ne renseigne pas sur ce qu'il faut entendre. Il est donc nécessaire de la mettre en perspective avec les épisodes du livre I. Cette fois, la polysémie de "columbinus " se prête à une troisième interprétation qui se combine avec les précédentes: "l'homme colombin" est, outre celui qui a la "simplicité de la colombe» et celui qui est habité par l'Esprit saint, celui que la colombe a "désigné» trois fois et à qui il est "voué».

39. I, 6 et II, 16 .

40. I, $6\left(\mathrm{f}^{\circ} 14 \mathrm{v}^{\circ}\right)$ : "Enimuero modeste circumspectus homo simplicitati columbine prudentiam serpentinam adequauerat $[. .$.$] ", adaptation de M t 10,16$ : "(...) estote ergo prudentes sicut serpentes et simplices sicut columbae".

41. I, 8 ( $\left.\mathrm{f}^{\circ} 21\right)$ : "Nec defuerunt iterum tres testes uenerabiles qui similiter ut prius in ipsa ordinatione super eum descendisse columbam et manisse uiderunt, et illud postea testimonio cui discredi non potest testificati sunt. Decebat nempe quatenus homo columbinus per columbam designaretur, et illi columbe dedicaretur, que teste Iohanne super Christem requieuit in Jordane".

42. I, 5 ( $\left.{ }^{\circ} 11\right)$ : « intima sancti Spiritus cohabitatio ». 


\section{Un art poétique}

C'est évidemment à l'intention d'un public averti que ce type de jeux littéraires sophistiqués quadrille l'ensemble de la Vita. Quoiqu'ils fonctionnent alors comme autant de clins d'œil érudits et appuyés, un auditoire moins choisi n'est pas pénalisé, au contraire même. Les acrobaties littéraires qu'exécute ici Baudri, sans jamais compromettre vraiment une compréhension globale du texte, participent pleinement à susciter l'admiration - qui n'a pas toujours besoin d'être clairvoyante - et l'éblouissement qui doivent s'emparer de quiconque est en présence d'une œuvre "de prix" réussie. Baudri nous donne d'ailleurs, transposées dans le domaine de l'orfèvrerie, des clefs pour comprendre ses partis-pris esthétiques. Dans le dernier chapitre du livre I ${ }^{43}$, il est en effet question d'une croix consacrée par Samson. Les frères du monastère où se trouve cet objet «de vile matière ${ }^{44}$ », quoique parfaitement conscients de la valeur de ce trésor légué par le saint, décident de donner une preuve sensible, tangible, de "leur dévotion ${ }^{45}$ " à Samson et de la transmettre: ils revêtent la croix de métaux précieux et la sertissent de gemmes. La conversion de cet objet intrinsèquement précieux mais sans valeur marchande en objet de prix ${ }^{46}$, en flattant les sens des spectateurs, leur font pressentir le caractère exceptionnel et sacré, non pas de la croix elle-même, mais de ce qu'elle évoque. On le voit, la démarche est analogue, même si l'objet travaillé diffère: les frères-orfèvres et l'archevêque-hagiographe poursuivent le même but d'édification spirituelle en s'appuyant sur des procédés d'ornementation semblables. De cela, nous trouvons la confirmation dans le prologue de la Vita sancti Hugonis, où Baudri explique qu'un matériau, si noble soit-il, ne saurait rester à l'état brut. Or, argent, ivoire, bois, marbre et autres gemmes doivent passer entre les mains d'un "polisseur" et «il n'en va pas différemment pour une histoire noble: si elle n'est pas lue avec recherche, elle s'avilit; si un style éloquent ne l'a pas colorée, elle déchoit de sa noblesse ${ }^{47}$ ".

Ainsi, quand il déclare dans son prologue avoir seulement voulu réaliser un texte de belle facture, seul capable de rendre compte du caractère sacré de son personnage, Baudri ne ment pas complètement. Il oublie seulement de dire que derrière les ornements de prix dont la "matière " est affublée se dissimule une conception différente du texte hagiographique elle-même adaptée à une nouvelle conception de l'édification.

43. I, 20 ( $\left.\mathrm{f}^{\circ} 58 \mathrm{sq}\right)$, intitulé "De cruce consecrata et ab homine malo furata ".

44. Idem : "crucem [...] materia uilem".

45. Idem : "[...] ut in hoc eorum deuotio dinosceretur".

46. Cette opération est si réussie qu'un voleur mal inspiré dérobe la croix. La colère posthume de Samson fond alors sur lui et le malheureux perd la vie dans des circonstances peu enviables.

47. P.L., t.162, prol., col. 1163. 


\section{L'édification efficace}

\section{Suppression des discours à vocation édifiante}

Le texte hagiographique avance donc masqué, séduisant sous les riches parures dont l'a revêtu Baudri: jamais il ne doit être pris en flagrant délit d'austérité, jamais il ne doit montrer un visage ennuyeux. C'est sans doute pourquoi les longs passages discursifs à vocation édifiante ${ }^{48}$ que l'hagiographe antérieur n'hésitait pas à greffer sur le récit ne subsistent qu'à l'état de vestiges. Baudri les a systématiquement, non pas purement et simplement supprimés, mais condensés en quelques mots intégrés au récit lui-même. Dans certains cas, il se contente de résumer en signalant le ton et le genre du propos. Ainsi «le bienheureux Samson les [ses auditeurs du moment] subjugua par un sermon édifiant ${ }^{49}$ " rend compte très succinctement d'un prêche d'une quinzaine de lignes ${ }^{50}$. Parfois même, Baudri élimine radicalement de son texte toute idée de discours. C'est ainsi qu'il conclut l'épisode de l'élection de Samson à la tête d'un monastère par «il [y] fut d'un grand secours pour les âmes des frères et de la population: toujours il se consacra spécialement tout entier à gagner leurs âmes ${ }^{51}$ ", quand dans la Vita carolingienne les propos de Samson pour persuader ses auditeurs de la nécessité de changer de vie se déploient sur plus d'une dizaine de lignes ${ }^{52}$. Il arrive enfin que le texte de Baudri joue de plusieurs procédés: la longue exhortation morale qu'adresse Samson à ses disciples avant de mourir est concentrée en quelques lignes par Baudri: «Samson, n’ignorant pas le jour de son départ, convoqua les frères pour les entretenir longuement et il les exhorta comme il faut en se référant aux dogmes de l'Évangile. Il leur recommandait de prendre en considération le jour de leur disparition et adjurait les assistants de ne pas tiédir dans la ferveur de leur religion: "Je marche sur la route de mes pères, mes très chers, mais, vous qui me survivrez, je vous recommande au Pasteur immortel ${ }^{53}$ ». Tous les ingrédients du texte initial s'y trouvent synthétisés: c'est en effet "longuement" (27 lignes) que Samson enjoint (nombreux impératifs et subjonctifs d'ordre, argumentation qui vise à persuader) à ses frères, citations de l'Évangile à l'appui, de vivre dans la perspective du jour de leur mort et surtout selon les préceptes chrétiens qu'il leur rappelle ${ }^{54}$. Ici le discours, bien qu'amplement

48. Nous regroupons sous cette appellation commode les prières, les exhortations et autres sermons. Prononcés le plus souvent par Samson, ces discours visent plus à édifier et instruire le lecteur / auditeur que les personnages du récit auxquels ils sont censés s'adresser.

49. II, 7 ( $\left.{ }^{\circ} 77\right)$ : «Beatus Sanson edificationis sermonem eis subiunxit ".

50. D.P.II, 7, p.128.

51. I, 13 ( $\left.{ }^{\circ} 38\right)$ : «[...] multum fratrum et populorum profuit animabus; cui lucro specialiter Sanson semper totus inuigilauit ".

52. D.P.I, 13, p.105.

53. II, 26 ( $\left.\mathrm{f}^{\circ} 103 \mathrm{v}^{\circ}-104\right)$ : "Diem migrationis sue Sanson non ignorans, fratres conuocans eos affatim allocutus est, et ex Euangeliorum dogmatibus ipsos luculenter exhortatus est. 
amputé, n'est pas entièrement sacrifié: le texte de Baudri conserve cette fois trace du discours lui-même en s'appuyant à la fois sur le discours rapporté («il adjurait les assistants de [...]») et une courte phrase au discours direct.

En allégeant ainsi son modèle, Baudri laisse clairement entendre que le procédé qui consiste à plaquer un message est grossier et surtout inopérant. C'est la raison pour laquelle il a nettoyé le texte de ces excroissances disgracieuses, et, ce coup de balai, en plus d'assainir efficacement le texte en contribuant à "l'embellir» selon les ambitions hagiographiques de Baudri, a l'avantage de privilégier le déroulement du récit: il met l'accent non sur ce qui se dit mais sur ce qui se fait, et plus précisément sur les actes de Samson et non sur le contenu de ses discours, option stylistique qui coïncide exactement avec la représentation que Baudri donne de Samson comme évangélisateur et prédicateur.

\section{Samson: un prédicateur en actes}

Le personnage est en effet peu bavard. Il n'en est pas pour autant aphasique, puisque, quoique sa pente naturelle le pousse à "se consacr[er] le plus souvent aux psaumes et au silence», "quand parfois il parl[e], son discours, assaisonné du sel de la religion, [a] toujours pour but d'édifier ${ }^{5}$ „. La remarque concerne ici Samson pendant ses années de formation, mais elle reste valable pour l'ensemble de sa carrière, à ce détail près que, dès l'instant où il est sacré évêque, il lui appartient d'édi-

Commendabat eis diem sue/[ $\left.f^{\circ} 104\right]$ dissolutionis, et adiurabat astantes ne aliquando tepescerent a feruore religionis. "Uiam patrum meorum, karissimi, ingredior, sed qui superstites nobis eritis, immortali Pastori uos commendo".

54. D.P.II, 26, p.148.9. Voici, dans son intégralité, le passage remodelé par Baudri: "Intervallo autem longaevo vetustatis senio fessus, cum diem transmigrationis imminere sibi sentiret convocatis ad se fratribus dixit: " Consideremus, fratres, quid simus, et quid erimus. Quid sumus, nisi homines? et unde homines, nisi de humo? et quid humus, nisi pulvis et cinis? Et quid erimus, nisi quod dictum est: "Cum apparuerit Christus, similes ei erimus", hoc est in aeternitate et immortalitate. Ideo abnegemus quod sumus, et incipiamus quod non sumus, Christo attestante qui ait: "qui vult venire post me, abneget semetipsum et tollat crucem suum, et sequatur me”. Ergo omne bonum a summo Deo sperandum est. Ideo cogitemus semper ultimum nostri obitus diem; ornemus finem nostrum, componamus vitam nostram. Et quantum incerti sumus de die nostri obitus, tantum parati simus in expectatione illius, ut quandocumque venerit, evigilet fides nostra, vigilantiae premium de domino receptura.

Consideremus et pensemus, quam districtus venturus est judex noster, qui non solum operum sed et cogitationum exactor apparebit. Sed ego precor Dominum ut ad bonum faciendum crescere vos faciat, et semper in melius vos proficere faciat. Et qui dedit vobis initium conversionis, ipse tribuere dignetur perseverantiam actionis, quia omne bonum in fine consistit. Non lassescat mens vestra, nec turbetur cor vestrum, viriliter agite, et omnia vestra in caritate fiant. Laborate in quantum potestis, quia non potestis quantum debetis. Ergo optandum est vobis cum summa intentione ad caeleste regnum festinare, in quo vocem desiderabilem audire mereamini, de qua Dominus dicit: "Venite, benedicti Patris mei, percipite regnum quod vobis paratum est ab origine mundi». Bene valeatis, Christo placeatis cui famulatis." 
fier à grande échelle. Dès lors, et c'est une innovation de Baudri conforme à l'esprit de la Réforme grégorienne, Samson devient l'archétype du prédicateur. En aucun cas, il ne s'adonne à de brillants exercices oratoires gratuits: c'est toujours sous le coup d'une nécessité impérieuse (sa fonction d'archevêque, un ordre angélique) ou sous des pressions diverses (on vient le chercher alors qu'il se terre, etc.) qu'il se résout à prendre la parole. Mais, une fois qu'il a commencé, il prononce avec facilité des sermons "éloquents", donne des conseils avisés et modérés, et surtout ses actes sont à la hauteur de ses discours, tant il est "prolixe en miracles ${ }^{56}$ ". Il remporte donc un fabuleux triomphe auprès des populations qu'il évangélise, mais le discours seul, si brillant et éloquent soit-il, est imparfait à susciter l'adhésion définitive à la foi: ce que dit Samson a beau être «miel et douceur inestimable", "la pieuse réalisation de ses actes" doit venir en renfort de "la douceur de ses propos ${ }^{57}$ ". Quand ce ne serait pas explicitement formulé ici et là dans le texte, le fait que la parole soit systématiquement inféodée à l'action suffirait à en témoigner. Chacun des discours de Samson, quel qu'en soit l'enjeu, doit être étayé par des actes, et, pas n'importe lesquels: de préférence des miracles spectaculaires, ainsi que Samson le dit lui-même. Alors qu'il rentre à Dol après un long séjour à la cour de Childebert ${ }^{58}$, il est interpellé par une femme qui lui réclame en ces termes de rendre à la vie son fils défunt: «Rends-moi, je te le demande, mon fils, toi qui m'a arraché mon époux (il faut dire que "son mari était entré en religion pendant un sermon du bienheureux maître»). Autrement je serai anéantie de douleur [...]. Fais aussi quelque chose ici dans ta patrie, toi, qui, à ce que nous avons entendu dire, a beaucoup agi en Francie $^{59}$ ». Aussitôt, Samson, incapable de "souffrir davantage le chagrin de cette femme ${ }^{60}$ " adresse à Dieu une prière: "Fais donc que revive la dépouille de ce défunt[...]. Il importe qu'en cette terre ton nom soit propagé, si tu as décidé de sauver ces populations. Ce peuple barbare, ce peuple insensible, il importe de le stimuler par des miracles inédits ${ }^{61}$ ". Samson reconnaît donc implicitement l'échec de la première campagne d'évangélisation (le mari de la femme éplorée a succombé aux charmes d'un sermon de Samson et, par ailleurs, on sait qu'au début de son installation à Dol, Samson sillonne les campagnes et cités alentours pour dis-

55. I, $8\left(\mathrm{f}^{\circ} 21 \mathrm{v}^{\circ}\right)$ : «Silentio et psalmodia plurimum occupatus erat. Si quando uero loquebatur, sermo eius sale religiosicatis conditus semper edificationis erat."

56. II, 1 (f 67 ): «Erat [...] dominus Sanson uultu iocundus, sermone disertus, assertione facundus, in consiliis perspicax et modestus, in miraculis copiosus[...]». On notera au passage qu'en plus de ses qualités oratoires et de sa générosité en matière de miracles, Samson dispose, ce qui ne gâche rien, d'un physique avantageux.

57. Idem ( $\left.\mathrm{f}^{\circ} 67 \mathrm{v}^{\circ}-68\right):$ "Mel [...] erat et quedam dulcedo inestimabilis quicquid dicebat, et dictorum dulcedinem pia operum exhibitio precipue commendabat."

58. Childebert $1^{\mathrm{er}}$ (511-558).

59. II, $15\left(\mathrm{f}^{\circ} 91 \mathrm{v}^{\circ}\right)$ "Redde, rogo, filium qui michi surripuisti maritum. Alioquin doloribus absorbebor [...] Fac et hic aliquid in tua patria, qui multa, ut audiuimus, operatus es in Francia".

60. Idem ( $\left.f^{\circ} 92\right)$ : "Non potuit sanctus diucius pati eius molestias". 
penser la parole divine, relevant pourtant ses propos de miracles ${ }^{62}$ ). Seule, d'après lui, une action d'éclat peut y remédier, et, effectivement, sitôt l'enfant ressuscité, les Bretons prennent conscience de la présence de Dieu à leurs côtés car "c'est désormais dans leur région, tout près, qu'ils le voient agir ${ }^{63}$ ". Dans le même ordre d'idées, alors que Samson ${ }^{64}$ entreprend, à l'instigation d'un tiers, "d'annoncer l'Évangile, escorté par le saint collège de ses frères ${ }^{65}$ " dans une région inculte et ignorante de Dieu ${ }^{66}$, il tombe en pleine "bacchanale» et décide aussitôt d'intervenir: «il se lanç[e] courageusement dans un prêche ${ }^{67}$ ", ce qui a pour seul résultat concret de déclencher la vindicte des participants. On en vient presque aux mains ${ }^{68}$, et, Samson ne doit son salut, celui des siens et celui des païens, qu'à la miséricorde divine: au moment crucial où enfle la colère de la foule qui "a presque le dessus ${ }^{69}$ ", un jeune noble tombe de cheval et en meurt. Samson qui a immédiatement compris le plan divin propose aux païens "insensés et entêtés ${ }^{70}$ " d'adorer son Dieu, en échange de quoi il rendra la vie au garçon. Et, bien sûr, ce qui devait arriver arriva: le mort est rendu à la vie, les païens détruisent leurs idoles et se font baptiser. Rien ne saurait mieux rendre compte de la faillite d'un discours désincarné et de la nécessité de l'actualiser: le discours est susceptible de n'être pas entendu et sujet à controverse, alors que les actes, dont la réalité est indiscutable, n'offrent aucune prise à la contestation et s'assurent un succès indéfectible.

\section{Un réalisme stratégique}

Baudri en tire les conclusions qui s'imposent pour sa version de la Vita Sansonis et c'est avec minutie et réalisme qu'il enrichit le récit de descriptions en apparence gratuites, en réalité stratégiques. Ainsi, il amplifie presque systématiquement les détails morbides, mais ceux-là seuls qui surviennent au cours des miracles accomplis par Samson: alors que l'épouse de Childebert ${ }^{71}$ a délégué un serviteur pour empoisonner Samson, celui-ci effectue le signe de croix sur le récipient qui contient le

61. Idem: "Fac igitur reuiuiscat gleba defuncti huius [...]. Oportet et in hac terra nomen tuum dilatari, si generationes istas saluare decruisti. Gentes iste barbare, gentes iste obdurate miraculorum nouitatibus oportet incitentur".

62. II, 1.

63. II, 15 ( $\left.\mathrm{f}^{\circ} 93\right)$ : "Sentiunt Britanni Deum sibi presentiorem quem iam in regione sua comminus cernunt operantem ".

64. I, 16.

65. I, $16\left(\mathrm{f}^{\circ} 44 \mathrm{v}^{\circ}-45\right)$ : "satagebat velociter ad Euangelium, sancto fratrum comitatus collegio".

66. Idem ( $\left.\mathrm{f}^{\circ} 44\right)$ : "in hac regione admodum a Deo barbara ".

67. Idem ( $\left.\mathrm{f}^{\circ} 45\right)$ : "[...] uidit ibi homines gentilium more bachanaliuiuentes, et quedam solemnia profanorum ritu paterno celebrantes.[...] Tactus dolore cordis intrinsecus heros uenerandus substitit, et eorum condolens errori in sermonem predicationis uiriliter irrupit".

68. Idem $\left(\mathrm{f}^{\circ} 45 \mathrm{v}^{\circ}\right)$ : "[...] ] alii conuiciis eum irritabant, alii pene in faciem eius irruebant".

69. Idem: "pene preualebat plebis cumulatio".

70. Idem ( $\left.\mathrm{f}^{\circ} 46\right)$ : "uecordes et obstinatos ". 
poison, lequel se répand sur la main du serviteur. Cet épisode relaté succinctement dans la Vita IIa où l'auteur se contente de signaler que la main est atteinte «jusqu'aux os» et que la victime «était éprouvé[e] au point de ne pouvoir plus se tenir debout, mais de pleurer amèrement ${ }^{72}$ " est considérablement développé par Baudri. Il s'attarde d'abord, avec un luxe de détails, sur la réaction de la main au contact de la "boisson mortifère ${ }^{73}$ ": «Le liquide pestifère humidifia la main de celui qui le présentait, lequel, comme s'il avait absorbé le poison malfaisant, sentit aussitôt qu'il avait été touché. En effet, elle commença à enfler immédiatement, et surtout, sur-le-champ, la peau se fendit en crevasses et le sang le plus interne laissa le poison s'associer à lui ${ }^{74}$ ». Puis il enchaîne en expliquant "scientifiquement" le phénomène: "La nature de cet homme s'indigna: le sang chaud ne pouvait supporter le poison glacial. Sa main et son bras fondaient et pourrissaient[...]", pour conclure sur les souffrances du malheureux serviteur qui «criait horriblement, comme s'il était possédé 75 ». De la même manière, il émaille les comptes rendus des punitions infligées à la reine et à la femme d'un seigneur de notations sanglantes. La première, bien que ses diverses tentatives pour éliminer Samson se soient soldées par de cuisants échecs - ce qui aurait dû lui servir de signal - s'acharne à s'attirer la foudre divine en tournant le dos à l'autel alors que Samson dit la messe: mal lui en prend puisque, nous dit le texte carolingien ${ }^{76}$, "comme on était arrivé à l'Agnus Dei, les yeux de la reine furent arrachés de sa tête, tombèrent à ses pieds sur la pierre, tout son sang jaillit à l'extérieur à l'endroit des yeux, et, bientôt [...] elle mourut de la mort la plus honteuse». Ici, Baudri précise seulement que "le sang s'épanchait et dégouttait sur la pierre" et souligne avec insistance la coïncidence frappante qui fait se confondre la prière du saint et la mort de la reine ${ }^{77}$. La seconde ${ }^{78}$, malgré un spectaculaire avertissement (la métamorphose de ses cochons en caprinés), s'entête également à braver l'homme de Dieu,

71. II, 5. Cette mala regina dont le nom n'apparaît ni ici ni dans les Vitae antérieures ne saurait être identifiée à l'épouse de Childebert $1^{\mathrm{er}}$, Ultrogothe, qui passe pour avoir été particulièrement pieuse. Il s'agit plutôt d'un stéréotype, sans doute inspiré de «méchantes reines" réelles, dont Brunehaut, la mère de Childebert II, honnie des milieux monastiques, serait le prototype (Cf. Pierre Flobert, op. cit., intro., p.28).

72. D.P.II, 5, p.126: "Et veneno effuso, in manus tenentis, videntibus cunctis, usque ad ossa crepuit [...] periclitabatur ille minister, qui poculum ei porrexerat, ita ut nullo modo stare posset, sed amare fleret".

73. II, 5 (f 74 ): "letalis potus ".

74. Idem: "Haustus pestifer manum porrigentis madefecit, qui continuo acsi uenenum hauserit malum infrictum sensit. Inchoauit enim ilico intumescere, immo et confestim cutis in rimula crepuit et sanguis interior uenenum sibi sociatum admisit."

75. Idem $\left(\mathrm{f}^{\circ} 74 \mathrm{v}^{\circ}\right)$ : "Indignata est hominis illius natura, et sanguis calidus uenenum frigidum ferre non poterat. Manus et brachium liquefiebat et tabescebat, et ille infelix horrendum clamabat et uelut insaniebat".

76. D.P.II, 9, p.130: "[...] cum ad Agnus Dei ventum est, oculi reginae capite suo avulsi sunt, et super petram ante pedes ejus ceciderunt, et totus ejus sanguis loco oculorum foras emanavit, moxque videntibus omnibus ibi adstantibus, antequam sanctus Sanson ultimam collectam compleret, foedissima morte defuncta est ". 
en voulant s'introduire dans l'oratoire du monastère de Pental interdit aux femmes. Semblable à celui qu'a subi plus haut la reine - à ceci près qu'elle n'en meurt pas -, le châtiment ne se fait pas attendre: «au moment de vouloir entrer dans l'église, elle tomba à terre sur la pierre de marbre resplendissante comme neige qui était devant le seuil de l'église; et aussitôt ses yeux tombèrent de sa tête sur la pierre, et leur sang, qui tomba sur la pierre, est visible, intact, aujourd'hui encore ${ }^{79}$ ». Là, l'inflation des précisions relatives à la mutilation est beaucoup plus évidente: "Les veines de ses yeux se rompirent, ses pupilles arrachées tombèrent instantanément sur la pierre, et ceux qui étaient là voyaient le sang dégoulinant s'épancher, comme si quelqu'un lui avait jeté la pierre à la figure», tout comme celles concernant la postérité du miracle: "Les signes de cet épisode sont encore observables sur le même caillou. Et en effet, voici qu'aujourd'hui certains endroits sur cette pierre blanche comme neige sont rouges: ce sont les endroits jadis aspergés par le sang de cette femme. Ainsi la pierre demeure intacte en témoignage, et les signes rouge-sang persistent en avertissement ${ }^{80}$ ».

\section{Actualisation}

Ces remaniements nous renseignent sur la manière dont Baudri conçoit qu'on doit prêcher et édifier. Quiconque s'adonne à cette activité - qu'il soit un saint mandaté par Dieu pour évangéliser des païens têtus ou convertir une cour royale, qu'il soit un archevêque raffiné du XII ${ }^{\mathrm{e}}$ siècle réécrivant la Vita de son saint patron - doit fournir des preuves concrètes de ce qu'il affirme. Ce qu'un Samson réalise avec des moyens exceptionnels, un Baudri le fait en renvoyant amplement à ces témoins irréfutables de la réalité passée que sont les "vestiges». On peut retrouver des traces matérielles qui attestent, pour l'éternité, l'intervention du saint: les gouttes de sang qui marquent, indélébiles, la pierre de Pental, un cours d'eau qui ruisselle à tel endroit ${ }^{81}$, une croix gravée sur un menhir ${ }^{82}$, etc. Mais l'on trouve encore le souvenir du passage de Samson dans la topo-

77. II, $9\left(\mathrm{f}^{\circ} 80 \mathrm{v}^{\circ}\right)$ : "Sanguis emanabat, et in petram distillabat. Antequam sanctus complesset ultimam collectam, regina morte horribili defuncta est. Completa est ergo insimul et Sansonis oratio, et diuina in filiam Eve ulcio (Avant que le saint n'eût achevé l'ultime oraison, la reine expira d'une mort horrible. Ainsi, c'est simultanément que la prière de Samson et la vengeance divine contre la fille d'Eve furent accomplies)».

78. II, 13.

79. D.P., p.135: "[...] dum in ecclesiam intrare vellet, super petram marmoream, sicut nix fulgentem, qui ante limen jacet ecclesiae, solo prostrata est; et statim oculi ejus de capite suo super petram ceciderunt, et sanguis eorum, qui super petram cecidit, sic usque hodie non deletus apparet".

80. II, 13 (f $\left.{ }^{\circ} 89\right)$ : «Disrupte sunt oculorum uene, et super lapidem auulse continuo ceciderunt pupille, et sanguinem irriguum, qui aderant uidebant emanare, tanquam si quilibet lapidem ei dedisset in capite. Cuius rei signa in eodem adhuc inspiciuntur silice. Rubent etenim ecce hodie quedam in ipso lapide niueo loca, quondam illo sanguine irrorata. Nam et lapis conseruatus manet in testimonium, et signa ruboris durant in monimentum ". 
nymie ${ }^{83}$, comme Baudri, plus sourcilleux que son prédécesseur en matière d'étymologie (souvent fantaisiste), l'explique à propos de Dol: «Saint Samson [...] voulut que le nom de cet endroit fût Douleur, évidemment en raison de la douleur que Privatus avait longtemps éprouvée [...]: actuellement, en langue plus moderne, on l'appelle Dol ${ }^{84}$ ". Mais ces signes concrets laissés à la postérité ne sont pas si nombreux: Baudri recourt donc aux subterfuges littéraires à sa disposition. Parfois, il se contente de tirer fidèlement les mêmes ficelles éprouvées que son prédécesseur, en amenant son auditoire à s'identifier à l'assistance qui entoure Samson. Dès que Samson est l'acteur principal d'un événement un peu conséquent, le texte signale l'attitude (stupéfaction, admiration, enthousiasme, effroi, etc.) du public, foule suffisamment neutre pour que l'assimilation fonctionne. Par ce jeu de mise en abyme, classique mais efficace, Baudri projette son auditoire réel dans le texte, lui permettant ainsi de mieux prendre part à tel ou tel miracle du saint. À cela s'ajoute un souci évident de réalisme. Visible dans les descriptions évoquées plus haut, il est également à l'œuvre dans certains discours dramatiques: mimétiques, dans leur forme même, de l'état d'esprit des personnages qui les prononcent, ils sont là pour rendre perceptible cet état d'esprit et le faire partager. Un exemple est particulièrement significatif à cet égard: au moment où Samson débarque en Bretagne, la Vita carolingienne indique seulement que la première personne rencontrée par le saint, Privatus, est en larmes et plongé dans la contemplation de la mer ${ }^{85}$. Baudri, lui, met véritablement en scène les larmes de Privatus: "[...] il se lamentait en sanglotant profondément. Il disait donc: "Oh! pourvu qu'il vienne! oh! pourquoi tardet-il? ah! quel misérable je suis! Quel infortuné! comme je suis indigne de consolations! Mon Dieu, que ta promesse soit vraie, et que mon péché ne soit pas un obstacle à Tes tendresses ${ }^{86}$ ». En substituant à la remarque allusive de la Vita IIa le discours d'un homme en proie à un chagrin immense, en lui conférant la parole, une parole cahotique, répétitive, larmoyante, il met l'accent sur la souffrance et les pleurs du personnage, il les donne à partager à son auditoire. Cet artifice, Baudri sait l'utiliser à

81. I, $17\left(\mathrm{f}^{\circ} 51 \mathrm{v}^{\circ}-52\right)$ : "Riuulus autem ille longis post temporibus ad perpetrati miraculi designationem fluere non cessauit [...]" et II, $10\left(\mathrm{f}^{\circ} 81 \mathrm{v}^{\circ}\right)$ : "[...] aquam eduxit, que usque hodie largissimo riuo defluit".

82. I, $16\left(\mathrm{f}^{\circ} 47 \mathrm{r}^{\circ}-\mathrm{v}^{\circ}\right)$ : «[... ] illa ymago crucis multo post tempore uisibilis et palpabilis perdurauit".

83. Outre l'exemple cité, on retrouve deux témoignages lexicaux des actes miraculeux de Samson: en II, 9 ( $\left.\mathrm{f}^{\circ} 80 \mathrm{v}^{\circ}-81\right)$ où Baudri indique l'origine nom Pental et en II, 21 ( $\left.\mathrm{f}^{\circ} 99 \mathrm{v}^{\circ}\right)$ où il explique l'origine du nom Rotinon.

84. II, $1\left(\mathrm{f}^{\circ} 66 \mathrm{v}^{\circ}\right)$ : "Nomen [...] loci illius dominus Sanson Dolor uocari uoluit, propter dolorem uidelicet quem Priuatus [...] diuturnum habuerat, quem modo lingua modernior Dolum uocat». La Vita IIa se contente d'indiquer que Samson "donna à l'endroit le nom de Dol à cause de la douleur de " Privatus (D.P.II, 1, p.121).

85. D.P.II, 1, p.102: "[...] sanctus Sanson vidit [...] virum, Privatum nomine, plorantem et ad mare semper respicientem". 
bon escient, ainsi que le montre un dernier exemple. Alors que le père de Samson est en proie à une maladie grave, des amis lui rendent visite et le pressent de songer à assurer le salut de son âme. La Vita IIa rapporte en ces termes la réaction d'Ammon: "Mais lui affirmait avec constance que jamais il ne goûterait la mort, que jamais il ne recouvrerait la santé, que jamais il ne communierait, s'il ne voyait pas de ses yeux son fils, saint Samson et, alors, grâce à lui il retrouverait, disait-il, la santé morale et physique ${ }^{87}$ ". Dans la version de Baudri, l'épisode est beaucoup plus impressionnant, parce qu'on y entend le malade parler, affirmer péremptoirement et, simultanément, se lamenter de l'absence de son fils: "On rapporte qu'il [Ammon] leur répondit: «Je ne guérirai pas de cette indisposition, je ne mourrai pas, si je ne vois pas mon fils très cher, Samson. Envoyez donc au plus vite pour savoir pourquoi, hélas, il ne vient pas! Faites venir auprès de moi la part la plus chère de mon âme ${ }^{88}$ ".

Conversion de passages narratifs un peu fades en discours pathétiques, réalisme de descriptions clés, mise en abyme: ce sont là quelquesuns des procédés littéraires qui, associés et conjugués à d'autres, sont capables de favoriser l'adhésion des auditeurs. Pour autant, ils sont insuffisants à réduire entièrement la distance infranchissable qui sépare un public, fût-il lettré, du XII ${ }^{\mathrm{e}}$ siècle de personnages, même historiques, dont les mœurs, les comportements ne sont plus toujours immédiatement compréhensibles.

\section{Rationalisation et normalisation}

\section{Escamotage d'éléments gênants}

Baudri en est bien conscient et, quoiqu'il se garde d'en souffler mot dans son prologue, il n'hésite pas à rendre Samson et ses contemporains familiers à son auditoire, et, par conséquent, à passer allègrement outre la lettre et l'esprit de son modèle. La solution la plus radicale trouvée par Baudri pour faciliter l'actualisation des personnages et du contexte est de supprimer tout ce qui est susceptible de se heurter à l'incompréhension ou à l'idéologie ambiante. Ainsi, la Vita IIa ${ }^{89}$ s'attarde longuement sur l'arrivée du jeune Samson au monastère d'Heldut et relate comment il n'éprouve aucun chagrin à voir ses parents s'éloigner, à l'inverse de n'importe quel enfant confronté à une situation similaire. Ce signe d'élection divine est d'ailleurs aussitôt confirmé, puisque Samson profite de sa

86. II, $1\left(f^{\circ} 61 v^{\circ}\right)$ : "[...] intimis singultibus eiulans. Dicebat igitur: « $O$ si ueniet! $O$ quid moratur? heu me miserum! heu me infelicem! heu me consolationibus indignum! Deus meus promissio Tua uera sit, neque peccatum meum Tuis obsit miserationibus ".

87. D.P.I, 9, p.94.

88. I, 9 ( $\left.{ }^{\circ} 24\right)$ : "Qui sic eis respondisse fertur: "Neque de hac inualitudine conualebo, neque moriar nisi prius filium meum carissimum Sansonem uidebo. Ut quid enim heuheu non uenit, mittite igitur quantocius, et anime mee portionem carissimam ad me accersite ". 
journée pour apprendre les lettres et leurs combinaisons, et qu'une semaine plus tard, il en maîtrise une lecture analytique. Baudri, lui, a soin d'omettre toute allusion aux circonstances de la séparation de Samson et de ses parents. Il ne pouvait sans doute pas accepter une telle indifférence chez son héros. En effet, quelques paragraphes plus tard ${ }^{90}$, Baudri se démarque de son modèle ${ }^{91}$ en inventant une scène d'adieux déchirante: au moment où Samson quitte la communauté d'Heldut pour gagner "un endroit plus secret ${ }^{92}$ ", "on [voit] les moines blêmir, on [voit] leur visage inondé de larmes, on les [voit] s'arracher les cheveux. Sangloter les emp[êche] de parler", Heldut lui-même "défaille" et Samson n'est pas en reste, puisqu'il s'éloigne finalement "non sans se lamenter grandement lui-même" et après s'être "séparé avec peine de son maître ${ }^{93}$ ». Signaler l'élection de Samson par sa froideur à l'égard de ses parents était, on le voit, incompatible avec cette représentation du saint. Quant à l'apprentissage-éclair de Samson, on le devine à peine derrière une évocation très globale, et très floue, des capacités exceptionnelles de l'enfant: "Il y avait chez Samson, outre une intelligence naturelle, une cohabitation intime avec l'Esprit Saint qui lui faisait connaître la science des lettres, et plus qu'à ses autres condisciples, lui dispensait une mémoire tenace. Heldut ne pouvait pas ne pas l'admirer, lui qui, dès ces jours de l'enfance, était poussé à augurer quelque chose de grand de Samson ${ }^{94}$ ". Il faut reconnaître que le passage relatif à l'apprentissage de Samson est particulièrement obscur dans la Vita IIa, et que la réalité évoquée est incompréhensible puisqu'il s'agit de pratiques inconnues de Baudri et même de son modèle qui semble recopier sans en rien comprendre sa propre source. Le silence de Baudri sur cet épisode est peutêtre l'aveu de son ignorance sur des pratiques pédagogiques anciennes (qu'on ne connaît encore que très mal aujourd'hui), mais, plus sûrement, et sans que ce soit paradoxal, il correspond au désir de normaliser Samson, dont l'enfance est déjà suffisamment exceptionnelle pour qu'on n'y insiste pas. Les enfances extraordinaires ne sont plus très en vogue au $\mathrm{XII}^{\mathrm{e}}$ siècle où apparaissent des saints dont la perfection n'est pas acquise

89. D.P.I, 5, p.87: "Parentes reversi sunt [...] Nullus more infantium post matrem patremve erat fletus, sed quasi a primis cunabilis suis ibi nutriretur, delectabiliter mansit. Mirum in modum sub una eademque die vicenas eleas tesserasque agnovit totas, nec opus fuit ei amplius monstrare. Et quod est mirabilius his omnibus, in una hebdomade omnium litterarum distinctiones per verborum conjunctiones, Deo revelante, potuit intelligere ".

90. I, 8 .

91. D.P.I, 8, p.93-94.

92. I, 8 (f 22$)$ : «locum secretiorem ».

93. I, $8\left(\mathrm{f}^{\circ} 22 \mathrm{v}^{\circ}\right)$ : “Videres monachos pallentes, lacrimis ora suffusos, cesarie disruptos. Singultus uerba prepediebat, [...] abbas [...] deficiebat. [...] Discessit igitur Sanson et ipse non sine eiulatu magno, et tandem uix auulsus est a magistro suo ".

94. I, 5 (f $\left.{ }^{\circ} 11\right)$ : "Aderat Sansoni preter ingenium naturale intima sancti Spiritus cohabitatio, que eius noticie litterarum scienciam suggerebat, et plus quam aliis condiscipulis tenacem memoriam ministrabat. Non mirari non poterat Heldutus, qui in ipsis puerilibus diebus de Sansone magnum quid auspicari cogebatur". 
et ostensible dès l'enfance. Baudri sait donc sacrifier aux exigences de son siècle des informations sinon essentielles du moins instructives. La formule originale qu'il a trouvée pour rendre compte de la disparition de l'abbé Piron en fait foi. Ce prêtre que Samson a rejoint après avoir quitté Heldut et dont "le nom était grand, la religion rigoureuse, la sainteté manifeste ${ }^{95}$ ", malgré ces qualités et d'autres encore (son cœur, comme celui de Samson, est un "cellier divin, où la plénitude de l'Évangile reposait ${ }^{96}$ "), a la malchance de mourir dans des circonstances peu canoniques. En état d'ébriété, il fait une chute mortelle dans le puits du monastère, ainsi que ne nous l'explique pas Baudri qui consent seulement à signaler qu'«il disparut d'une mort insolite ${ }^{97}$ ". L'allusion, pour le moins concise et énigmatique, ne laisse rien transparaître des indications données par la version carolingienne ${ }^{98}$, mais elle a l'avantage incontestable de conserver à Piron son statut de saint, alors qu'au même moment un Guibert de Nogent ${ }^{99}$, indigné de cette mort effectivement «insolite», lui dénie toute espèce de sainteté.

\section{Réinterprétations}

Baudri n'abuse pas de cette méthode qui consiste à effacer les éléments gênants: il préfère les réinterpréter. C'est ainsi qu'il lit 100 " quodam librario ${ }^{101}$ ", expression désignant l'homme de grande renommée à qui Ammon et son épouse vont s'adresser pour résoudre les problèmes de stérilité d'Anna, non pas comme l'évocation d'“un certain copiste» aux pouvoirs plus ou moins magiques mais comme une allusion à «un certain Librarius». Il est peu plausible que Baudri ait fait la confusion machinalement, étant donné que son modèle, quand il introduit le nom d'un personnage, le fait explicitement précéder de "nomine". Le passage de la fonction (librarius) au nom propre (Librarius) doit plutôt être mis sur le compte du désir qu'a Baudri d'élucider le texte de son prédecesseur. Il lui permet de concilier les données du texte carolingien qui attribue trop généreusement les fonctions de " magister 102 " et de prophète à un copiste et de rendre le personnage plus vraisemblable. C'est probablement dans le même ordre d'idée qu'il fait subir à un personnage singulier de la Vita

95. I, 8 ( $\left.{ }^{\circ} 23\right):$ "[...] quidam presbiter magni nominis, ardue religionis, predicande sanctitatis".

96. Idem $\left(\mathrm{f}^{\circ} 23 \mathrm{v}^{\circ}\right)$ : «Pectora quippe eorum quedam diuina apotheca erant, ubi Euangelii plenitudo quiescebat" .

97. I, $10\left(\mathrm{f}^{\circ} 31 \mathrm{v}^{\circ}\right):$ «[...] Piro morte inopina obiit ».

98. D.P.I, 10, p.101: «Nam non longe post in eodem monasterio res inopinata gesta est. Idem Piro in tenebras nocte per ineptam ebrietatem in claustra monasterii deambulans solus in puteum valde vastum cecidit, et a fratribus jam emortuus abstractus est, et in illa nocte obiit.» (D.P., p.101).

99. De sanctis et eorum pignoribus I, 1, P.L. 156, col. 614, cité par Bernard MERDRIGNAC, Les Vies de saints bretons durant le haut Moyen Âge, Ouest France université, 1993.

100. I, 2.

101. D.P., p.83. 
II ${ }^{103}$ une métamorphose importante. Alors que Samson et son compagnon de voyage, un diacre, traversent une forêt, ils entendent une voix qui retentit atrocement ${ }^{104}$ et tombent nez à nez avec une "theomacha" qui commence par tuer le diacre avant de prendre la fuite. Samson la somme d'arrêter sa course et de décliner son identité ou, plutôt, de révéler sa nature. Comme elle est "irrécupérable ${ }^{105}$ " et irrémédiablement vouée au mal, il prie Dieu de la faire mourir, ce qui lui est aussitôt accordé. Dans la version carolingienne, la "théomaque" apparaît comme une vieille sorcière aux cheveux blancs, hirsute et en haillons. À la main, elle tient ce qui ressemble à un trident. Quand Samson l'interroge pour en savoir plus, elle dit être une "théomaque ${ }^{106}$ " - ce qui n'est pas très éclairant - et indique qu'elle est la seule de sa race à vivre dans la forêt. Ses huit sœurs et sa mère vivent dans une autre forêt. Quant à elle, elle est arrivée dans ce bois en suivant son mari, lequel est mort et, dit-elle, "à cause de cela, [elle] ne peu[t] quitter cette forêt ${ }^{107}$ ". La suite de son entretien avec Samson nous apprend qu'elle est incapable de rendre la vie au frère qu'elle a tué car elle "ne peu[t] pas faire le bien ${ }^{108}$ ". Dans la version de Baudri, Samson et le diacre entendent bien d'abord «une voix très effrayante ${ }^{109}$ ", mais rapidement, cette voix perd toute dimension humaine pour se muer en un «bruit aussi insolite qu'effroyable», un "vacarme effroyable ${ }^{110}$ ». Survient alors «l'esprit immonde» qui a "revêtu une forme féminine mais hirsute et hérissée», qui est "déformé en une monstruosité digne de sa laideur ${ }^{111}$ ». C'est donc cette "apparition excessivement mauvaise», "noire, difforme, terrible» qui "agite à la main un épieu" et "terrasse" le diacre avant de s'enfuir ${ }^{112}$. Sur l'injonction de Samson, elle s'arrête et lui donne, quand il l'interroge une réponse sybilline: «Je suis théomaque parmi les théomaques, si par hasard tu as entendu parler de la théomachie ${ }^{113}$ ». Alors, Samson déclare: «Puisqu'assurément tu t'es obstiné de toute ta méchanceté dans le mal, que tu y es voué, au nom du Seigneur, n'outrage plus quiconque, n'apparaîs plus à personne, ne fais de mal à personne ${ }^{114}$ ", ce qui a le don de la faire disparaître. Le passage du féminin au masculin montre bien que

102. D.P., p.83-4: 5 occurrences, contre 2 occurrences pour "librarius ".

103. I, 9. D.P., p.96-97.

104. Idem, p.96: "[...] dum irent, orantes per vastissimam silvam durissimam audierunt vocem, juxta illos terribiliter strepitantem".

105. Idem, p.97: "irremediabilis ».

106. Idem, p.96: "Theomacha sum».

107. Idem, p.97: "et propter hoc recedere de hac silva non possum ".

108. Idem: "Bonum enim facere nequeo".

109. I, $9\left(\mathrm{f}^{\circ} 25 \mathrm{v}^{\circ}\right)$ : «uocem audierunt dirissimam ".

110. Idem : "sonitu [...] insolito et horrendo " et ( $\left.\mathrm{f}^{\circ} 26\right)$ : " horrendo strepitu".

111. Idem $\left(\mathrm{f}^{\circ} 26\right)$ : "[...] uidet Sanson spiritum immundum in formam muliebrem sed hirsutam, sed hispidam transformatum, et in condignam turpitudinem deformatum ".

112. Idem: "Persona autem illa nequissima dyaconum fugientem percussit, prostrauit. [...] Videbat eam Sanson imperteritus ultra quam credi potest, nigram deformem, terribilem, uenabulum manu uibrantem ". 
Samson n'interpelle pas la "théomaque» en tant que manifestation féminine mais en tant qu'esprit ou apparition: toute humanité lui est déniée; elle n'est plus une sorcière, avec des parents et une histoire, elle est une "illusion". Baudri investit le mot "theomacha" d'un sens précis (une variété d'esprits capables de revêtir une apparence humaine) et construit toute la scène à partir de la manière dont il comprend ce terme. Son propos en devient très cohérent, jusque dans les moindres détails: ainsi, la disparition de la "théomaque" est conforme à sa nature ambiguë, puisqu'on ne sait si elle "s'évanouit" (comme un esprit, ce qu'elle est) ou si elle "meurt" (comme un humain, ce dont elle a pris l'apparence) ${ }^{115}$. D'ailleurs, pour qu'il n'y ait aucune confusion possible, Baudri prend à parti son auditoire et lui indique comment comprendre l'épisode: "Quant à nous, nous n'ignorons pas que les esprits malins sont de semblables illusions, à qui est parfois octroyée la capacité d'être vues et de faire le $\mathrm{mal}^{116}$ ".

\section{Commentaires explicatifs}

Ce type d'intervention directive, plus ou moins explicative, a pour but de légitimer le récit. Baudri circonscrit la lecture de l'épisode et prévient toute investigation de la part de l'auditoire. C'est encore plus flagrant quand il s'agit de l'épisode capital relatant la consécration épiscopale ${ }^{117}$ de Samson. Cette fois, Baudri ne remet pas en cause le témoignage de l'hagiographe carolingien ${ }^{118}$ et répète fidèlement ses indications dont voici résumées les grandes lignes: trois évêques, dont Dubricius, doivent, à l'occasion de festivités, ordonner trois nouveaux évêques et il s'avère qu'il leur manque un troisième postulant. Pendant la nuit, Samson apprend en songe qu'il est celui-là, ce dont Dubricius est également averti par le même canal. À la lumière des deux songes, il apparaît que la volonté divine a désigné Samson et il est alors promu évêque. Bien que le mode de consécration soit surprenant, le contresens commis par son prédécesseur «induit sans doute en erreur par la latinité confuse de la Vita Ia qu'il remaniait ${ }^{119}$ " ne semble pas avoir posé problème à Baudri: il reprend l'assertion selon laquelle "ce mode de consécration (que trois évêques soient toujours consacrés par trois évêques) s'était enraciné dans ces contrées et la coutume d'une antique simplicité était observée comme on

113. Idem $\left(\mathrm{f}^{\circ} 26 \mathrm{v}^{\circ}\right)$ : "De theomachis una sum theomacha, si forte theomachiam audisti."

114. Idem: "Quando quidem in malo obstinatus es nequissime et destinatus, in nomine Domini nulli amplius iniuriam facias, nemini ulterius appareas, nemini noceas ".

115. Idem: "uel euanuit uel dicessit".

116. Idem ( $\left.\mathrm{f}^{\circ} 26\right)$ : "Nos autem tales prestigias spiritus malignos non nescimus, quibus et quedam facultas ut aliquando uideantur et noceant permittitur".

117. I, $14\left(\mathrm{f}^{\circ} 38-40\right)$.

118. D.P.I, 14, p.105-6. 
l'a dit 120 ». Apparemment, l'éloignement géographique et temporel suffit à authentifier cette pratique et Baudri y souscrit sans sourciller. Ce qui le contrarie davantage, c'est que seules des voix surnaturelles élisent Samson. C'est pourquoi il vient interrompre la narration par un long commentaire en forme de plaidoyer: "Que personne ne murmure qu'une telle consécration ne fut pas canonique: celle que Dieu choisit de préférence et approuva, nous, nous la déclarons hardiment canonique et catholique. Et l'Esprit saint n'est pas soumis aux institutions des hommes, mais tantôt par une orientation qu'on ne peut comprendre, il approuve les lois des hommes, tantôt, dénonçant l'hébétude de notre sens, il dispense avec bonté ce qui nous sera utile sans tenir compte des mortels ${ }^{121}$ ». Sans doute s'agit-il d'éviter que l'auditoire ne s'interroge sur la canonicité et, par conséquent, la validité de l'accès à l'épiscopat de Samson. Il faut qu'elles soient bien établies afin que son œuvre ultérieure, son archiépiscopat et celui de ses successeurs ne soient pas sujets à caution. Cette fois, en choisissant de suivre scrupuleusement la version proposée par son modèle, Baudri s'est condamné à le commenter et à en extraire le sens caché, afin d'éviter que le doute, ou même la curiosité, ne s'empare de l'auditoire. Le texte ne doit jamais prêter le flanc à d'éventuelles critiques ni soulever des questions, ce qui nuirait à une réception efficace du récit. Chaque fois que le récit risque d'achopper, Baudri s'efforce d'aplanir le texte, soit qu'il insère des commentaires explicatifs ou des justifications, soit qu'il en modèle le contenu pour l'adapter aux exigences de l'auditoire.

Ce souci de normalisation et d'explication qui se conjugue avec un travail stylistique et formel évident montrent bien que Baudri ne s'est pas «borné à corriger les fautes de style et de langue qui s'étaient glissées dans" son modèle ni contenté d'en "gloser [le] texte quand il était obscur ${ }^{122}$ ". Une véritable réflexion sur le statut, le mécanisme et la fonction du texte hagiographique parcourt la Vita Sansonis que donne Baudri. En l'assimilant à un objet qu'il faut embellir pour rendre sensible et permanent son caractère vénérable et admirable auprès du plus grand

119. CARREE, André et MERDRIGNAC, Bernard, «La Vie latine de saint Lunaire», Britannia monastica, C.I.R.Do.Mo.C, 1991, p.44.

120. I, 14 ( $\left.\mathrm{f}^{\circ} 39\right)$ : "Is etenim modus episcopos consecrandi illis in partibus inoleuerat, ut scilicet tres semper a tribus consecrarentur, et antique simplicitatis consuetudo taliter seruaretur".

121. I, $14\left(\mathrm{f}^{\circ} 39 \mathrm{r}^{\circ}-\mathrm{v}^{\circ}\right)$ : « Talem autem consecrationem nemo susurret non fuisse canonicam, quoniam quam Deus preelegit, et approbauit, nos audenter profitemur canonicam et catholicam. Neque siquidem Spiritus sanctus humanis subiectus est institu/tis[f $\left.f^{\circ} 39 v^{\circ}\right]$, sed quodam suo incomprehensibili moderamine interdum leges hominum approbat, interdum nostri sensus hebitudinem redarguens, quedam ultra mortalium considerationem nobis profutura clementer dispensat ". 
nombre, Baudri indique que toute Vita, et en particulier les Vitae qu'il compose, est à la fois œuvre d'art et outil de propagande idéologique.

Que s'y greffe une propagande éventuellement plus personnelle n'a rien de surprenant, surtout quand celui dont on exalte les vertus est aussi celui dont on doit assumer l'héritage contesté, comme c'est le cas ici. Il est d'ailleurs difficile de croire que Baudri, si épris de littérature soit-il, ait déployé toutes les ressources de son art de manière désintéressée - mais ceci outrepasse le cadre du présent article.

\section{RÉSUMÉ}

Baudri de Bourgueil a composé la Vita sancti Sansonis dans le premier tiers du $\mathrm{XII}^{\mathrm{e}}$ siècle, alors qu'il était archevêque de Dol. Ce texte a longtemps été considéré, sur la foi des déclarations préliminaires de l'auteur, comme un remaniement stylistique dénué d'intérêt autre que littéraire. La comparaison entre la Vita de Baudri et sa source, une Vita carolingienne, et l'examen attentif des modalités de réécriture révèlent cependant que ces prétentions de réfection purement formelle et stylistique masquent une véritable réflexion sur la nature et la finalité du texte hagiographique. Comme Baudri se fait ici l'hagiographe de celui qui passe pour être le fondateur de la métropole et qui en fut le premier archevêque, il est permis de penser que ses préoccupations esthétiques, morales et religieuses ne sont pas seules à donner du sens à sa version de la Vita sancti Sansonis.

\section{ABSTRACT}

The Vita sancti Samsonis was written by Baudri de Bourgueil in the first third of the XII century, when he was the archibishop of Dol. According to the own introduction of the author, it has been regarded for a long time as a stylistic rewriting without any major interest. However a comparison between the Vita by Baudri and his source, a carolingian text, a closer examination of the way it was written show that this formal and stylistic rewritten work hides deep thought on hagiographic texts function and aims.

122. FAWTIER, R., La vie de saint Samson, essai de critique hagiographique, Bibl. de l'École des Hautes Études (fasc. 197), éd. Honoré Champion, 1912, p.17. 